\title{
Calidad de vida en el trabajo y su relación con el engagement
}

\author{
Claudia Alejandra Toscano-del Cairo; Juan Javier Vesga-Rodríguez; Bertha Lucía Avendaño-Prieto
}

How to cite this article:

Toscano-del Cairo, C.A., Vesga-Rodríguez, J.J. \& Avendaño-Prieto, B.L. (2020). Quality of life at work and its relationship with engagement. Acta Colombiana de Psicologia, 23(1), 128-137. doi: http://www.doi.org/10.14718/ACP.2020.23.1.7

Recibido, octubre 31/2018; Concepto de evaluación, febrero 12/2018; Aceptado, abril 11/2019

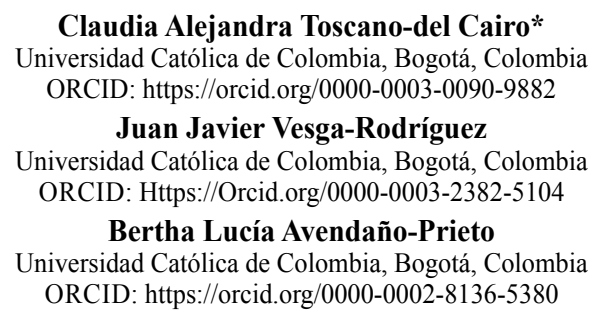

Resumen

\begin{abstract}
Con el objetivo de explorar la relación funcional entre el engagement y la calidad de vida en el trabajo (CVT), en el presente estudio se utilizó una estrategia de investigación asociativa con 221 empleados de una institución de educación superior en Colombia, quienes accedieron a participar de manera voluntaria. Para evaluar la CVT se aplicó el instrumento "Perfil de Calidad de Vida Laboral", desarrollado por Gómez (2010), conformado por 39 ítems que evalúan ocho dimensiones; mientras que, para evaluar el engagement, se utilizó la adaptación realizada por Ospina y Delgado-Abella (2014) del UWES (Utrecht Work Engagement Scale), desarrollada por Schaufeli y Bakker (2004), que consta de 17 ítems que evalúan tres dimensiones: vigor, dedicación y absorción. Como resultado, se halló una asociación significativa entre las distintas variables del estudio a partir de un modelo de ecuaciones estructurales; y se encontró evidencia sobre las dimensiones de la CVT que más aportan para su configuración, además de las diferencias por sexo en las puntuaciones de las variables de estudio. Palabras clave: engagement, calidad de vida en el trabajo, ecuaciones estructurales.
\end{abstract}

\section{Quality of life at work and its relationship with engagement}

\begin{abstract}
In order to explore the functional relationship between Engagement and Quality of Life at Work, an associative research strategy was used. 221 employees of a higher education organization in Colombia participated, who voluntarily agreed to be part of the study. To evaluate the quality of life at work, the instrument "Profile of Quality of Work Life" developed by Gómez (2010) was applied, which consists of 39 items that evaluate 8 dimensions of this construct. To evaluate the variable Engagement, the adaptation made by Ospina and Delgado-Abella (2014) of the UWES - Utrecht Work Engagement Scale developed by Schaufeli and Bakker (2004) was used, which consists of 17 items that evaluate three dimensions: vigor, dedication and absorption. There was a significant association between the study variables. The relationship and the adjustment are presented in a model of structural equations. Evidence was found about the dimensions of the QWL that contribute most in the configuration of this variable, and the differences by sex in the punctuations of the research variables.

Key words: Engagement, Quality of Life at Work, Structural Equations.
\end{abstract}

Av. Caracas n. 46-72, Bogotá. D. C., Colombia. Tel.: (57-1) 327-7300. catoscanocanal@gmail.com, jjvesga@ucatolica.edu.co 


\section{Introducción}

En la actualidad, la gestión de personal en las organizaciones demanda importantes retos para los directivos de diversas empresas en el mundo, debido principalmente a las transformaciones que han ocurrido en las últimas cuatro décadas en distintos ámbitos con respecto a las dinámicas sociales, en general, y a la estructuración y gestión de las organizaciones, en particular. Tales transformaciones han provocado que los líderes empresariales afronten un ambiente de negocios altamente dinámico, caracterizado por una permanente innovación tecnológica, un desvanecimiento de las fronteras entre las industrias y los sectores de negocios, cambios en el comportamiento del consumidor, y escasez de talento, entre otros factores que afectan de manera importante el desarrollo de sus empresas (Boston Consulting Group, 2014).

En este contexto, las organizaciones enfrentan cambios importantes tanto en la gestión de personal como en el lugar de trabajo y el mundo laboral (Deloitte, 2017), por lo que la Gerencia de Recursos Humanos (GRH) se ha transformado también y ha pasado de la administración de personal a convertirse en una función estratégica de las organizaciones, donde las políticas y prácticas de gestión deben tener el doble propósito de, por una parte, contribuir a la productividad y competitividad de las organizaciones, $y$, por otra, procurar el desarrollo y bienestar de los trabajadores independientemente del tipo de contratación que tengan. En este sentido, una estrategia clave de la GRH que contribuye al logro de la competitividad de las organizaciones en el contexto actual de trabajo es la "experiencia del empleado", ya que "[e]l rediseño del lugar de trabajo, programas de bienestar y los sistemas de productividad laboral se están convirtiendo en parte de los temas mandatorios para RH" (Deloitte, 2017, p. 7).

Así, en un contexto de transformaciones tanto en la sociedad como en el mundo del trabajo, la GRH también requiere de cambios de paradigmas en la psicología aplicada a la gestión de personas en las organizaciones, con los cuales se busque, principalmente, pasar de un modelo centrado en la enfermedad a uno focalizado en la salud y el bienestar (Salanova \& Martínez, 2005). En este orden de ideas, una perspectiva que toma fuerza en la actualidad para la GRH con una orientación hacia el desarrollo, el compromiso, la salud y el bienestar, es la psicología positiva, la cual, en el campo de las organizaciones, se ha denominado "psicología organizacional positiva", y se define como "el estudio científico del funcionamiento óptimo de la salud de las personas y de los grupos en las organizaciones, así como la gestión efectiva del bienestar psicosocial en el trabajo y del desarrollo de las organizaciones saludables" (Salanova, Martínez \& Llorens, 2014, p. 23).

Desde esta perspectiva, como mencionan Wilson, DeJoy, Vandenber, Richardson y McGrath (2004):

[u]na organización saludable es aquella que se caracteriza por esfuerzos intencionales, sistemáticos y colaborativos para maximizar el bienestar y la productividad de los empleados mediante la provisión de empleos significativos y bien diseñados, un ambiente de apoyo social y organizacional, y oportunidades accesibles y equitativas de carrera y el enriquecimiento trabajo-familia (p. 567).

Así, uno de los propósitos fundamentales de la psicología positiva $-\mathrm{y}$ característica relevante de las organizaciones saludables - es el esfuerzo por el mejoramiento continuo de la calidad de vida en el trabajo (CVT) (Salanova, Martínez \& Llorens, 2014); concepto que implica aspectos tanto subjetivos como objetivos asociados con la solución de necesidades del trabajador y su percepción sobre las prioridades de estas.

Específicamente, la CVT se define como un "concepto multidimensional que se basa en la satisfacción, a través del empleo, de una amplia gama de necesidades personales, ubicándolo como la clave para la realización personal, familiar y social y como medio para preservar la economía y la salud" (González, Hidalgo \& Salazar, 2007, p. 121), que implica tanto acciones para la procura por el diseño de condiciones saludables del entorno de trabajo, como el interés por identificar y buscar satisfacer las necesidades personales de los empleados.

En un nivel similar al interés por la CVT, los líderes organizacionales requieren y se interesan por mantener altos niveles de compromiso por parte de sus empleados, ya que "[1] a cultura y el compromiso son aspectos clave en la experiencia del empleado, y las organizaciones líderes están expandiendo su foco para incluir desde el primer contacto de una persona con un empleador hasta la jubilación y más allá" (Deloitte, 2017, p. 7).

Adicional a esto, una perspectiva particular, pero más amplia que el estudio del compromiso en el contexto de la psicología positiva, es la investigación en el concepto de engagement, el cual se ha definido como "un estado psicológico positivo caracterizado por altos niveles de energía y vigor, dedicación y entusiasmo por el trabajo, así como la total absorción y concentración en la actividad laboral" (Salanova \& Schaufeli, 2004, p. 109). De este modo, tanto la CVT como el engagement han demostrado ser variables importantes que afectan el desempeño laboral y que tienen un impacto tanto en la productividad como en la competitividad de las organizaciones (García \& Forero, 2016; Yongxing, Hongfei, Baoguo \& Lei, 2017). 
130

Dado que estas dos variables son clave en la gestión de las organizaciones, es importante estudiar la relación entre ellas, pues identificar posibles relaciones permite comprender de manera más definida las características de las interacciones entre sus dimensiones y proporcionar orientaciones basadas en la evidencia para el desarrollo de estrategias dirigidas a lograr altos niveles de compromiso en los empleados a través de programas de mejoramiento de la CVT.

En función de esto, el presente estudio se planteó con el objetivo de identificar la relación entre la CVT como variable predictora y el engagement como variable criterio. De esta manera, se espera aportar elementos basados en la evidencia para orientar el desarrollo de procesos que mejoren la CVT en las organizaciones y faciliten mejores niveles de engagement. Asimismo, se espera poder identificar el aporte de la variable sexo en esta relación, puesto que algunos estudios sugieren la existencia de importantes diferencias en la relación de esta variable tanto con la CVT (Gómez \& Ponce de León, 2010) como con el engagement (Salas-Vallina \& Alegre, 2017).

\section{Método}

\section{Diseño}

Se utilizó una estrategia asociativa, cuyo objetivo es explorar la relación funcional entre variables. Específicamente, se empleó un diseño predictivo transversal que permitió analizar la relación entre la CVT y el engagement (Ato, López \& Benavente, 2013).

\section{Participantes}

Se utilizó un muestreo no probabilístico en el que se seleccionó a 221 empleados de una institución de educación superior (IES) que accedieron a colaborar voluntariamente. Como criterios de inclusión se tuvo en cuenta que fueran trabajadores contratados por la compañía y cuyo tiempo de vinculación fuera superior a tres meses. Se excluyeron trabajadores con tiempo de vinculación inferior a tres meses, aprendices SENA, y pasantes o practicantes universitarios.

En la Tabla 1 se encuentra la distribución porcentual de las variables sociodemográficas. Específicamente, el $59.5 \%$ de los participantes eran mujeres y el $40.5 \%$ eran hombres; el promedio de edad total fue de 40.6 años; en cuanto al nivel de estudios, predominó el personal con posgrado completo, con una participación del $29.5 \%$; y, respecto al estado civil, el mayor porcentaje lo presentaron las personas casadas, con un $36.4 \%$ de participación.

\section{Instrumentos}

Por una parte, para la variable CVT se aplicó el instrumento "Perfil de calidad de vida laboral", desarrollado por Gómez (2010), cuya validación se hizo con una muestra de 250 empleados de tres empresas de los sectores público y privado ubicadas en la ciudad de Bogotá, Colombia. En el estudio original, el instrumento obtuvo un índice de confiablidad de .88 (modelo de Rasch), mientras que, en el presente estudio, dicho índice fue de 915 . Este instrumento fue desarrollado a partir de las dimensiones propuestas por Walton (1973) y está conformado por 39 ítems que evalúan ocho dimensiones: compensación y beneficios $(\mathrm{CyB})$, condiciones del ambiente y del trabajo (CAT), naturaleza de la tarea (NT), desarrollo y seguridad laboral (DSL), democracia organizacional (DO), derechos fundamentales (DF), equilibrio laboral (EL), e impacto social (IS). La escala de respuesta tiene cuatro niveles: "totalmente en desacuerdo", "parcialmente en desacuerdo", "parcialmente de acuerdo" y "totalmente de acuerdo".

Y, por otra parte, para la variable engagement se utilizó la adaptación realizada por Ospina y Delgado-Abella (2014) del UWES (Utrecht Work Engagement Scale), desarrollado por Schaufeli y Bakker (2004), que consta de 17 ítems que evalúan tres dimensiones: vigor, dedicación y absorción. Acá, la escala de respuesta consta de siete niveles, con una puntuación de 0 a 6 , donde $0=$ nunca (ninguna vez); $1=$ casi nunca (pocas veces al año); 2 = algunas veces (una vez al mes o menos); 3 = regularmente (pocas veces al mes); $4=$ bastantes veces (una vez por semana); $5=$ casi siempre (pocas veces por semana); y $6=$ siempre (todos los días). Dicha adaptación se hizo en una muestra de profesores y funcionarios administrativos de una universidad en la ciudad de Bogotá y obtuvo un índice de confiabilidad (alfa de Cronbach) de .902. En la presente investigación, el alfa fue de .87 .

\section{Procedimiento}

Inicialmente, se obtuvo la autorización por parte de la Dirección de la División de Gestión del Talento Humano de la IES, y, posteriormente, tras ubicar los instrumentos en la herramienta "Formularios de Google", se le envió un correo electrónico al personal administrativo para que respondiera a los cuestionarios de manera virtual, mientras que con el personal del área de servicios generales se aplicaron los cuestionarios de manera presencial, al convocar reuniones grupales para su aplicación - todos los participantes 
Tabla 1.

Distribución porcentual de variables sociodemográficas

\begin{tabular}{|c|c|c|c|}
\hline Variable & Categoría & $\mathrm{n}$ & $\%$ \\
\hline \multirow{2}{*}{ Sexo } & Hombres & 89 & 40.5 \\
\hline & Mujeres & 131 & 59.5 \\
\hline Edad & \multicolumn{3}{|c|}{$\mathrm{M}=40.6$ años; DT $=10.63$ años; Mínimo $=20 ;$ Máximo $=63}$. \\
\hline \multirow{11}{*}{ Último nivel de estudio alcanzado } & Primaria incompleta & 6 & 2.7 \\
\hline & Primaria completa & 5 & 2.3 \\
\hline & Bachillerato incompleto & 6 & 2.7 \\
\hline & Bachillerato completo & 17 & 7.7 \\
\hline & Técnico/Tecnológico incompleto & 12 & 5.5 \\
\hline & Técnico/Tecnológico completo & 49 & 22.3 \\
\hline & Profesional incompleto & 15 & 6.8 \\
\hline & Profesional completo & 33 & 15.0 \\
\hline & Postgrado incompleto & 11 & 5.0 \\
\hline & Postgrado completo & 65 & 29.5 \\
\hline & No contestó & 1 & .5 \\
\hline \multirow{6}{*}{ Estado civil } & Casado & 80 & 36.4 \\
\hline & Divorciado & 9 & 4.1 \\
\hline & Separado & 7 & 3.2 \\
\hline & Soltero & 76 & 34.5 \\
\hline & Unión libre & 46 & 20.9 \\
\hline & Viudo & 2 & .9 \\
\hline
\end{tabular}

accedieron voluntariamente a formar parte del estudio-. Por último, tras la recolección y depuración de los datos, como siguiente paso se inició el análisis de resultados correspondiente con el software SPSS y con el AMOS.

\section{Aspectos éticos}

Los empleados de la IES firmaron el consentimiento informado, donde se constata que recibieron información suficiente sobre los cuestionarios y el procedimiento. Por otra parte, los empleados que participaron en el estudio - en los términos de la ley 1581 de 2012 - manifestaron a los investigadores su autorización, de manera libre, previa y voluntaria, para dar el tratamiento de los datos suministrados por cada uno en el formulario.

\section{Análisis de datos}

Primero, con el propósito de describir las características laborales de los participantes, se realizó la distribución porcentual de las variables relacionadas con el trabajo y se identificaron los descriptivos de cada una de las dimensiones de los dos instrumentos aplicados; posteriormente, se comparó por sexo las medias de cada una de las de las dimensiones de los instrumentos utilizados, puesto que esta variable aportó al modelo encontrado; y, por último, se empleó el modelo de ecuaciones estructurales para el análisis de la relación entre las variables, aunque, como estas no se ajustaron a una distribución normal, se utilizó el método de distribución asintóticamente libre, empleado particularmente cuando las variables del modelo son categóricas (Browne, 1984; Lara, 2014).

Para el análisis de bondad de ajuste del modelo se siguieron las indicaciones señaladas en la literatura (Pilatti, Godoy \& Brussino, 2012) y se utilizaron los estadísticos chi-cuadrado (CMIN), la razón de chi-cuadrado sobre los grados de libertad (CMIN/DF), el índice de ajuste comparativo (CFI), el índice de bondad del ajuste global (GFI), el índice no normalizado de ajuste o Tucker Lewis (TLI), el IFI (Incremental Fit Index) y el error cuadrado de aproximación a las raíces medias (RMSEA).

El estadístico chi-cuadrado indica el ajuste absoluto del modelo, pero es muy sensible al tamaño de la muestra, por consiguiente, se utilizó la razón de chi cuadrado sobre los grados de libertad, donde los valores inferiores a tres indican un buen ajuste. Por otra parte, los índices CFI y GFI varían entre cero y uno — cero indica ausencia de ajuste y uno un ajuste óptimo-; y los valores de .95 o superiores son considerados excelentes, mientras que valores superiores a .90 sugieren un ajuste aceptable del modelo a los datos. 
132

También, el índice RMSEA es considerado óptimo cuando sus valores son de $.05 \mathrm{o}$ inferiores y aceptables en el rango de .05 a .08; mientras que el TLI es considerado aceptable con puntuaciones mayores o iguales a .90 (Escobedo, Hernández, Estebané \& Martínez, 2016). El IFI es indicativo de buen ajuste cuando cuenta con valores superiores a .90 (Leal-Costa, Tirado-González, Rodríguez-Marín \& van der Hofstadt-Román, 2016).

Finalmente, el modelo propuesto implica la CVT como variable predictora y su relación con el engagement como variable criterio, así como la posible relación entre algunas de las dimensiones constitutivas de las dos variables de estudio y las variables sociodemográficas y laborales.

\section{Resultados}

Con la finalidad de examinar la relación funcional entre el engagement y la CVT, inicialmente se realizó el análisis descriptivo de las variables relacionadas con el trabajo y de las dimensiones de las variables CVT y engagement; posteriormente, se establecieron las diferencias de medias por sexo; y, por último, se realizó el análisis correlacional para las variables objeto de estudio con el modelo de ecuaciones estructurales.
Con respecto a los datos descriptivos de las variables relacionadas con el trabajo, cabe mencionar que, del total de la muestra - 221 participantes-, el $85.9 \%$-que corresponde a 189 personas - respondió los cuestionarios en forma virtual, mientras que el porcentaje restante lo hizo en forma presencial; además, que los participantes llevaban en promedio 10.4 años trabajando en la organización; que el mayor porcentaje de los cargos desempeñados se distribuyó entre auxiliares (32.7\%) y profesionales (33.2\%); que una mayor proporción se encontraba en áreas administrativas $(71.36 \%)$; y que el $80.5 \%$ tenía un contrato a término indefinido. En la Tabla 2 se presenta la distribución porcentual de estas variables.

Ahora bien, frente a las dimensiones de las variables, la puntuación media más alta en la variable CVT la obtuvo la dimensión IS (3.58, de una puntuación máxima de 4); mientras que, para la variable engagement, la puntuación media más alta la obtuvo la dimensión de dedicación (5.25, de una puntuación máxima de 6). En la Tabla 3 se presentan los descriptivos de cada una de las dimensiones de los dos instrumentos aplicados.

Por otra parte, con respecto a las diferencias por sexo, se hizo la comparación de las medias de cada una de las dimensiones en las dos variables, con lo que se encontró que, de la variable CVT solo había diferencias estadísticamente significativas en la dimensión de naturaleza de la tarea (NT)

Tabla 2.

Distribución porcentual de las variables relacionadas con el trabajo

\begin{tabular}{|c|c|c|c|}
\hline Variable & Categoría & $\mathrm{n}$ & $\%$ \\
\hline \multirow{4}{*}{ Cargo desempeñado } & Auxiliar, asistente administrativo, asistente técnico. & 72 & 32.7 \\
\hline & Jefatura (tiene personal a cargo). & 39 & 17.7 \\
\hline & Operario, operador, ayudante, servicios generales. & 36 & 16.4 \\
\hline & Profesional, analista, técnico, tecnólogo. & 73 & 33.2 \\
\hline \multirow{5}{*}{ Nivel del cargo } & Dirección especializada. & 14 & 6.36 \\
\hline & Operativo. & 37 & 16.82 \\
\hline & Profesional. & 79 & 35.91 \\
\hline & Técnico. & 85 & 38.64 \\
\hline & No contestó. & 5 & 2.27 \\
\hline \multirow{3}{*}{ Unidad } & Académica. & 57 & 25.91 \\
\hline & Administrativa. & 157 & 71.36 \\
\hline & No contestó. & 6 & 2.73 \\
\hline \multirow{4}{*}{ Tipo de contrato } & Temporal de un año o más. & 21 & 9.55 \\
\hline & Temporal de menos de un año. & 19 & 8.64 \\
\hline & Término indefinido. & 177 & 80.45 \\
\hline & No contestó. & 3 & 1.36 \\
\hline
\end{tabular}

Tiempo de trabajo en la organización

$\mathrm{M}=10.4$ años; DT $=8.73$ años; Mínimo $=0$ años; Máximo $=37$ años. 
Tabla 3.

Descriptivos de cada una de las dimensiones de los dos instrumentos aplicados

\begin{tabular}{lcc}
\hline \multicolumn{1}{c}{ Dimensiones de los instrumentos } & Media & Desviación estándar \\
\hline Perfil de Calidad de Vida Laboral & & .69 \\
Compensación y beneficios (CyB) & 2.84 & .40 \\
Condiciones del ambiente y del trabajo (CAT) & 3.49 & .45 \\
Naturaleza de la tarea (NT) & 3.50 & .59 \\
Desarrollo y seguridad laboral (DSL) & 2.97 & .67 \\
Democracia organizacional (DO) & 2.87 & .52 \\
Derechos fundamentales (DF) & 3.47 & .55 \\
Equilibrio laboral (EL) & 3.34 & .43 \\
Impacto social (IS) & 3.58 & \\
UWES & & .63 \\
Vigor & 5.12 & .70 \\
Dedicación & 5.25 & .64 \\
Absorción & 4.94 & \\
\hline
\end{tabular}

Tabla 4.

Diferencias de medias por sexo en los puntajes obtenidos en la dimensión NT

\begin{tabular}{ccccccc}
\hline Dimensión & Sexo & $\mathrm{n}$ & $\mathrm{M}$ & $\mathrm{DE}$ & $\mathrm{t}$ & $\mathrm{p}$ \\
\hline \multirow{2}{*}{ NT } & Hombre & 89 & 3.59 & .41 & \multirow{2}{*}{2.47} & .01 \\
& Mujer & 131 & 3.44 & .46 & & \\
\hline
\end{tabular}

Tabla 5 .

Diferencias de medias por sexo en los puntajes obtenidos en las dimensiones vigor y dedicación

\begin{tabular}{llcccc}
\hline \multicolumn{1}{c}{ Variable } & \multicolumn{1}{c}{ Sexo } & M & DE & t & Sig. bilateral \\
\hline \multirow{2}{*}{ Vigor } & Hombre & 5.12 & .49 & \multirow{2}{*}{2.40} & .01 \\
\multirow{2}{*}{ Dedicación } & Mujer & 5.29 & .47 & & \multirow{2}{*}{.00} \\
& Hombre & 5.27 & .56 & \multirow{2}{*}{2.79} & .00 \\
\hline
\end{tabular}

Nota. $M=$ media, $D E=$ desviación estándar.

(véase Tabla 4), y que la media de los hombres fue superior a la de las mujeres; mientras que, de la variable engagement, solo hubo diferencias estadísticamente significativas en las dimensiones de vigor y dedicación (véase Tabla 5), y, contrario a lo sucedido con la variable CVT, la media de las mujeres fue superior a la media de los hombres.

Por último, para el análisis de la relación entre las variables se hizo uso del modelo de ecuaciones estructurales —en la Tabla 6 se presentan algunos indicadores de ajuste del modelo-, en donde se contó con que los índices de bondad de ajuste obtenidos con el AMOS fueron la razón entre el valor de chi-cuadrado $\left(\chi^{2}\right)$ y el número de grados de libertad correspondientes $\chi^{2} / \mathrm{gl}$, cuyo valor fue de 1.471 ; y que el índice global de ajuste GFI fue de .98 , el índice de ajuste comparativo CFI, de .93, y el RMSEA, de .05, con un intervalo de confianza entre .021 y .071. Cabe mencionar que el CFI compara la mejora en el ajuste del modelo en cuestión a un modelo nulo, en el que todos los ítems son independientes - no se permiten factores comunes-, y que este índice es de los más utilizados debido a que es poco sensible al tamaño de la muestra (Sternberg, Prieto \& Castejón, 2000).

Específicamente, en la Figura 1 se muestra el diagrama de la estructura con los respectivos indicadores de las relaciones entre las variables y sus dimensiones - con base en las puntuaciones promedio (PROM) de cada una- Como

Tabla 6.

Evaluación del ajuste del modelo analizado

\begin{tabular}{cccccccc}
\hline CMIN & DF & CMIN/DF & GFI & IFI & TLI & CFI & RMSEA \\
\hline 72.083 & 49 & 1.471 & .98 & .93 & .90 & .93 & $.049[.021 ; .071]$ \\
\hline
\end{tabular}


se puede observar, en cuanto a los coeficientes de regresión entre las variables latentes y las dimensiones observadas, los primeros ocho valores $(.74 .75, .79, .77, .80, .69, .68 \mathrm{y}$ .74) correspondieron a la influencia de la variable latente CVT sobre las dimensiones observadas CyB, CAT, NT, DSL, DO, DF, EL e IS; donde sobresale que la influencia que ejerce la variable latente sobre las dimensiones observadas indica que cuando la CVT aumenta una unidad, las variables compensación y beneficios (CyB) e impacto social (IS) aumentan en proporciones de .74. Como todos los coeficientes de regresión tienen signo positivo, la relación entre las variables latentes (CVT y engagement) y las dimensiones observadas es directa.

Aunque el objetivo general fue identificar la relación entre la CVT y el engagement en una organización de educación superior en Colombia, en el modelo de ecuaciones estructurales se incluyeron para su análisis las variables sociodemográficas - sexo, edad, escolaridad y estado civil- y las variables laborales - cargo desempeñado, nivel del cargo, unidad, tipo de contrato y tiempo de trabajo en la organización-; sin embargo, la única variable cuyo aporte resultó significativo fue el sexo, con un valor $\mathrm{p}=.023$ y un peso de regresión de .80 . Adicional a esto, entre la CVT y el engagement se encontró un efecto positivo de la primera variable sobre la segunda, dado que la relación entre estas dos tiene una carga de valor estimado de .61, con un nivel de significancia de .04; en otras palabras, el peso de regresión para la CVT en la predicción del engagement es significativamente diferente de cero en el nivel de .05 (dos colas).

Con base en los resultados, se estableció que existe relación directa entre la CVT y el engagement, con un efecto positivo de la primera variable sobre la segunda, dado que la relación entre estas dos variables tiene una carga de valor estimado de .61 (valor recomendado $\geq .07$ ) (Escobedo, Hernández, Estebané \& Martínez, 2016). Igualmente, se estableció que las dimensiones que más aportan a la CVT son DO (.80), MNT (.79), DSL (.77), CAT (.75), CyB (.74) y MEL (.74).

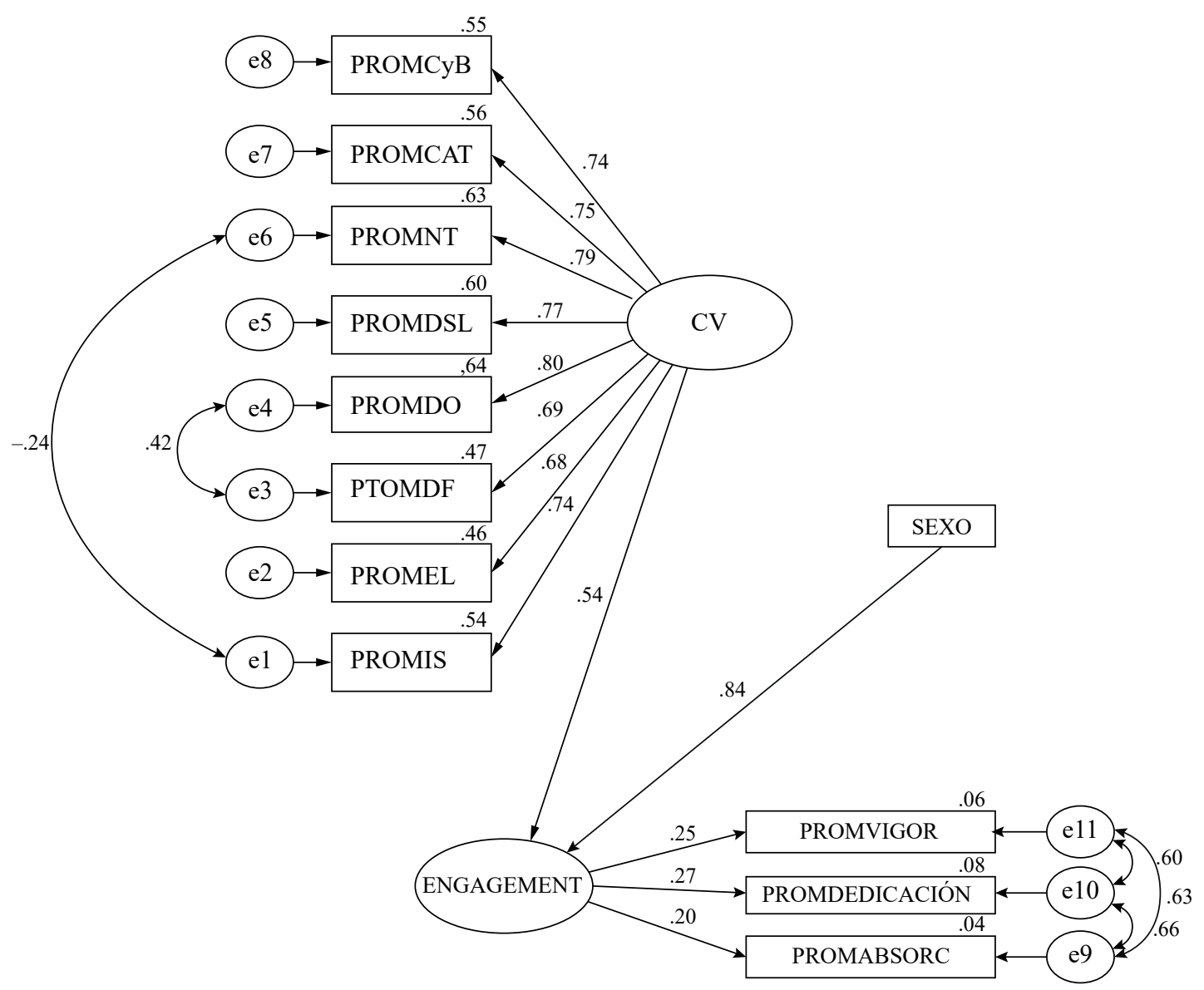

Figura 1. Relación entre las variables CVT y engagement con las estimaciones estandarizadas. 


\section{Discusión}

El propósito principal de esta investigación fue identificar la relación entre la CVT y el engagement en una IES en Colombia. El modelo planteado sugería una relación entre estas dos variables y los resultados confirman esa hipótesis. En tal sentido, el estudio ofrece información pertinente para la GRH en las organizaciones de trabajo.

En un mundo laboral cada vez más complejo y variado, en el que múltiples factores pueden incidir en la manera como los empleados se relacionan con y perciben su trabajo, la CVT juega un papel preponderante en su nivel de compromiso con el trabajo y con la organización, por lo que el conocimiento de las dinámicas y composición de factores asociados con la CVT le permite a los directivos de las organizaciones - y, en particular, a la GRH - dirigir sus esfuerzos de gestión focalizándolos en la intervención de aquellos aspectos que mayor impacto tienen en el desempeño organizacional. A partir de esto, el presente estudio ha permitido identificar una relación directa de incidencia de la CVT sobre el engagement, así como algunas características clave acerca de la relación de las dimensiones con las respectivas variables.

En cuanto a la CVT, las puntuaciones medias de las distintas dimensiones se encuentran por encima del $50 \%$ (véase Tabla 3), lo que permite evidenciar que, en general, los empleados de la organización perciben muy buenas condiciones de CVT. Sin embargo, los coeficientes de regresión en la relación de las dimensiones con la variable (véase Figura 1) ponen de manifiesto que, de las ocho dimensiones, seis de ellas — con puntuaciones superiores a .70 - son las que más aportan a la variable. Esto evidencia que las dimensiones con mayor carga en la configuración de la CVT son las relacionadas con aspectos personales - como desarrollo y seguridad laboral (DSL) y naturaleza de la tarea (NT) - , con aspectos del contexto de trabajo e institucional - como condiciones del ambiente y del trabajo (CAT) e impacto social (IS) - y las relacionadas con condiciones de justicia y equidad - como compensación y beneficios (CyB) y democracia organizacional (DO) -

En este sentido, los factores del contexto de trabajo e institucional, las condiciones que facilitan el desarrollo personal y profesional de los empleados, así como la equidad y la justicia organizacional (Colquitt, Conlon, Wesson, Porter \& Ng, 2001) se constituyen en componentes fundamentales de la CVT.

Cabe mencionar que la justicia organizacional es un concepto de las ciencias del comportamiento que se refiere a la percepción de equidad en el tratamiento recibido por los empleados en una organización (Hosmer \& Kiewitz, 2005), y dado que, tal como lo han sugerido Yadav y Yadav (2016), el activo más importante de cualquier organización son sus empleados - así como la razón de la ventaja competitiva en cualquier sector empresarial-, por lo tanto, la principal preocupación de cualquier organización debe centrarse en los esfuerzos por lograr satisfacción, compromiso, elevados niveles de desempeño y bienestar en el trabajo; en este sentido, cualquier iniciativa para fortalecerlos debe tener siempre como marco la justicia organizacional.

Con respecto al engagement, las tres dimensiones obtuvieron puntuaciones medias-altas (véase Tabla 3), lo cual muestra niveles elevados de engagement en los empleados de la organización. Como lo refieren Salanova \& Schaufely (2004), esta variable es lo opuesto al burnout — un estado mental persistente negativo, caracterizado por un agotamiento emocional que afecta negativamente la CVT y el desempeño en el trabajo- $-\mathrm{y}$, en este orden de ideas, altos niveles de engagement actúan como factores protectores en el desarrollo de estados de burnout.

En cuanto a las diferencias significativas por sexo encontradas en la variable CVT para la dimensión de naturaleza de la tarea (NT) (véase Tabla 4) - dimensión que permite identificar la percepción de los empleados acerca de la manera en que los cargos que desempeñan, además de que posibilita "la utilización y el desarrollo de sus capacidades profesionales y personales, autonomía, el uso de habilidades múltiples, la ejecución de tareas globales y no segmentadas, información clara acerca de las actividades que realiza y posibilidades de planeación" (Gómez, 2010, p. 119) - , la puntuación media fue más alta por parte de los hombres (3.59), con diferencias significativas respecto de la media en las mujeres; lo cual evidencia la percepción de una mayor articulación entre las capacidades personales y el puesto de trabajo, así como una mayor autonomía y variedad en el trabajo para los participantes hombres.

Por otra parte, con respecto a las diferencias significativas por sexo encontradas en la variable engagement (véase Tabla 5), principalmente en las dimensiones de vigor - que hace referencia a elevados niveles de energía y resistencia mental en el trabajo - y dedicación —entendida como el entusiasmo y el orgullo por el trabajo- (Salanova \& Schaufeli, 2004), la puntuación media más alta de correspondió a las mujeres (5.29 en vigor y 5.48 en dedicación), con diferencias significativas frente a la media en los hombres, lo cual evidencia mayores niveles de engagement para las mujeres en estas dos dimensiones.

También, con el modelo de ecuaciones estructurales - presentado en la Figura 1-se logró identificar el efecto directo de la CVT sobre el engagement; hallazgo que sugiere que los esfuerzos ejecutados por las organizaciones en virtud del mejoramiento de la CVT generan efectos positivos en 
los niveles de engagement. En este sentido, se puede decir que los empleados estarán más dispuestos a comprometerse con su trabajo si perciben que la organización para la cual trabajan provee condiciones adecuadas para su CVT, especialmente en aspectos que favorecen su desarrollo y la justicia organizacional. Al respecto, algunos investigadores, como Rodríguez, Martínez y Salanova (2014) han evidenciado relaciones similares, sobre todo con el engagement como variable mediadora en la relación entre la justicia organizacional y los comportamientos de ciudadanía organizacional, ya que "cuando los empleados son tratados de forma justa, equitativa, con dignidad y respeto, estos se podrán sentir más engaged con sus trabajos" (p. 970).

Con estos resultados en cuenta, las acciones dirigidas a mejorar la CVT producen entonces efectos en doble vía: por una parte, son un interés que manifiesta la organización por los propios empleados que se relaciona con los beneficios que reciben los mismos empleados directamente; $\mathrm{y}$, por otra, el mayor involucramiento y compromiso de los empleados en su trabajo redundará en mejores niveles de desempeño y productividad, con beneficios para la organización. De este modo, los resultados obtenidos muestran soluciones coherentes con la teoría, ya que "la investigación científica ha puesto de manifiesto como posibles causas del Engagement: los recursos laborales (ej.: autonomía, apoyo social) y personales (ej.: autoeficacia)" (Salanova \& Schaufeli, 2004, p. 119).

Finalmente, un hallazgo importante de esta investigación se relaciona con el efecto de moderación de la variable sexo en la relación entre la CVT y el engagement, sabiendo que "[u]na variable moderadora es una variable que altera la magnitud y/o la dirección de la relación existente entre $X$ e Y" (Ato \& Vallejo, 2011, p. 554). En este sentido, en el modelo de ecuaciones estructurales propuesto, el efecto directo de la CVT sobre el engagement aumenta considerablemente cuando se introduce la variable sexo en el modelo, lo cual sugiere que la relación entre estas dos variables se ve afectada por el sexo. Esto implica que se deben tener en cuenta las diferencias por sexo al momento de diseñar los programas de las organizaciones dirigidos a mejorar la CVT con el propósito de aumentar los niveles de engagement, debido a que las mismas acciones no impactarán por igual a hombres y mujeres.

Futuras investigaciones deberían contemplar la posibilidad de llevar a cabo estudios cualitativos o mixtos que ofrezcan - a través de narrativas, por ejemplo- datos textuales que permitan comprender aspectos de la subjetividad de los trabajadores implicados en las acciones del trabajo relacionadas con su percepción sobre la CVT y el engagement, o con respecto a las diferencias de sus efectos dependiendo del sexo de los empleados. Igualmente, se abre el horizonte para revisar la relación de la influencia de las variables que componen la CVT y el engagement por sectores de la economía, para así trazar con más precisión programas de calidad de vida que impacten de manera eficaz y oportuna el compromiso de los trabajadores, focalizando en este punto los recursos e inversiones de los programas de gestión humana.

\section{Referencias}

Ato, M., López, J., \& Benavente, A. (2013). Un sistema de clasificación de los diseños de investigación en psicología. Anales de Psicología, 29(3), 1038-1059. doi: 10.6018/ analesps.29.3.178511

Ato, M., \& Vallejo, G. (2011). Los efectos de terceras variables en la investigación psicológica. Anales de Psicología, 27(2), 550-561. Recuperado de https://revistas.um.es/ analesps/article/view/123201/115851

Boston Consulting Group. (2014). Creating People Advantage 2014-2015. Boston: The Boston Consulting Group, Inc.

Browne, M. W. (1984). Asymptotically distribution-free methods for the analysis of covariance structures. British Journal of Mathematical and Statistical Psychology, 37(1), 62-83. doi: 10.1111/j.2044-8317.1984.tb00789.x

Colquitt, J. A., Conlon, D. E., Wesson, M. J., Porter, C., \& Ng, K. Y. (2001). Justice at the Millennium: A Meta-Analytic Review of 25 Years of Organizational Justice Research. Journal of Applied Psychology, 86(3), 425-445. doi: 10.1037//0021-9010.86.3.425

Deloitte. (2017). 2017 Deloitte Global Human Capital Trends. Reescribiendo las reglas para la era digital. Recuperado de https://www2.deloitte.com/gt/es/pages/human-capital/arti cles/introduction-human-capital-trends-2017.html

Escobedo, M. T., Hernández, J. A., Estebané, V., \& Martínez. G. (2016). Modelos de ecuaciones estructurales: Características, fases, construcción, aplicación y resultados. Ciencia \& trabajo, 18(55), 16-22. doi: 10.4067/ S0718-24492016000100004

García, M., \& Forero, C. (2016). Calidad de vida laboral $\mathrm{y}$ la disposición al cambio organizacional en funcionarios de empresas de la ciudad de Bogotá-Colombia. Acta Colombiana de Psicología, 19(1), 79-90. doi: 10.14718/ ACP.2016.19.1.5

Gómez, C. A. (2010). Diseño, construcción y validación de un instrumento que evalúa la calidad de Vida laboral percibida en organizaciones colombianas, desde la teoría de respuesta al ítem. Psychologia. Avances de la disciplina, 4(1), 113124. doi: 10.21500/19002386.1163

Gómez, C. A., \& Ponce de León, E. (2010). Modelo integrativo de la calidad de vida laboral percibida a partir de la inseguridad laboral, la jornada laboral y el salario, para hombres y mujeres trabajadores de empresas de la ciudad de Bogotá 
en diferentes rangos de edad. Psychologia. Avances de la disciplina, 4(2), 113-129. doi: 10.21500/19002386.1149

González, R., Hidalgo, G., \& Salazar, J. G. (2007). "Calidad de vida en el trabajo": un término de moda con problemas de conceptuación. Psicología y Salud, 17(1), 115-123. doi: 10.25009/pys.v17i1.746

Hosmer, L. T., \& Kiewitz, C. (2005). Organizational justice: a behavioral science concept with critical implications for business ethics and stakeholder theory. Business Ethics Quarterly, 15(1), 67-91. doi: 10.5840/beq20051513

Lara, A. (2014). Introducción a la Ecuaciones estructurales en Amos y R. Recuperado de http://masteres.ugr.es/ moea/pages/curso201314/tfm1314/tfm-septiembre1314/ memoriamasterantonio_lara_hormigo/!

Leal-Costa, C., Tirado-González, S., Rodríguez-Marín, J., \& van der Hofstadt-Román, C. J. (2016). Psychometric properties of the Health Professionals Communication Skills Scale (HP-CSS). International Journal of Clinical and Health Psychology, 16(1), 76-86. doi: 10.1016/j.ijchp.2015.04.001

Ospina, C. A., \& Delgado-Abella, L. (2014). Adaptación lingüística y cultural del Cuestionario Utrecht Work Engagament (UWES) a la población colombiana. Documento no publicado. Universidad Católica de Colombia.

Pilatti, A., Godoy, J. C., \& Brussino, S. A. (2012). Análisis factorial confirmatorio del cuestionario de expectativas hacia el alcohol para adolescentes (CEA-A). Acta Colombiana de Psicología, 15(2), 11-20. Recuperado de https://edito rial.ucatolica.edu.co/ojsucatolica/revistas_ucatolica/index. php/acta-colombiana-psicologia/article/view/262/pdf $\% 20$ Art $\%$ C3\%ADculo\%201

Rodríguez, R. L., Martínez, M., \& Salanova, M. (2014). Justicia organizacional, engagement en el trabajo y comportamientos de ciudadanía organizacional: una combinación ganadora. Universitas Psychologica, 13(3), 961-974. doi: 10.11144/Javeriana.UPSY13-3.joet

Salanova, M., \& Martínez, I. L. (2005). Psicología Organizacional Positiva. En F. J. Palací, Psicología de la Organización (pp. 349-376). Madrid: Pearson.

Salanova, M., \& Schaufeli, W. (2004). El Engagement de los empleados: un reto emergente para la dirección de los recursos humanos. Estudios Financieros, 261, 109-138. Recuperado de http://www.want.uji.es/download/el-enga gement-de-los-empleados-un-reto-emergente-para-la-direccion-de-los-recursos-humanos/
Salanova, M., Martínez, S. M., \& Llorens, S. (2014). Una mirada más "positiva" a la salud ocupacional desde la Psicología Organizacional Positiva en tiempos de crisis: aportaciones desde el equipo de investigación WoNT. Papeles del Psicólogo, 35(1), 22-30. Recuperado de http://www.pape lesdelpsicologo.es/pdf/2318.pdf

Salas-Vallina, A., \& Alegre, J. (2017). The role of gender, age, job level and job security on physician's engagement in Spanish public hospitals. Universia Business Review, (56), 36-51. doi: 10.3232/UBR.2017.V14.N4.02

Schaufeli, W., \& Bakker, A. (2004). Utrecht Work Engagement Scale. Preliminary Manual-Version 1.1. Recuperado de https://www.wilmarschaufeli.nl/publications/Schaufeli/ Test\%20Manuals/Test_manual_UWES_English.pdf

Sternberg, R. J., Prieto, M. D., \& Castejón, J. L. (2000). Análisis factorial confirmatorio del Sternberg Triarchic Abilities Test (nivel-H) en una muestra española: resultados preliminares. Psicothema, 12(4), 642-647. Recuperado de http://www. psicothema.com/english/psicothema.asp?id=384

Walton, R. E. (1973). Quality of Working Life: What Is It? Sloan Management Review, 15(1), 11-21.

Wilson, M., DeJoy, D., Vandenber, R., Richardson, H., \& McGrath, A. (2004). Work characteristics and employee health and well-being: Test of a model of healthy work organization. Journal of Occupational and Organizational Psychology, 77(4), 565-588. Recuperado de https://www. deepdyve.com/lp/wiley/work-characteristics-and-employee-health-and-well-being-test-of-a-9QThZOqOsC

Yadav, L. K., \& Yadav, N. (2016). Organizational Justice: An Analysis of Approaches, Dimensions and Outcomes. NMIMS Management Review, 31, 14-40. Recuperado de https://pdfs.semanticscholar.org/9e61/c0de4b29882c10e97ee67f04584f6682c6a0.pdf

Yongxing, G., Hongfei, D., Baoguo, X., \& Lei, M. (2017). Work engagement and job performance: the moderating role of perceived organizational support. Anales de psicologia, 33(3), 708-713. doi: 10.6018/analesps.33.3.238571 